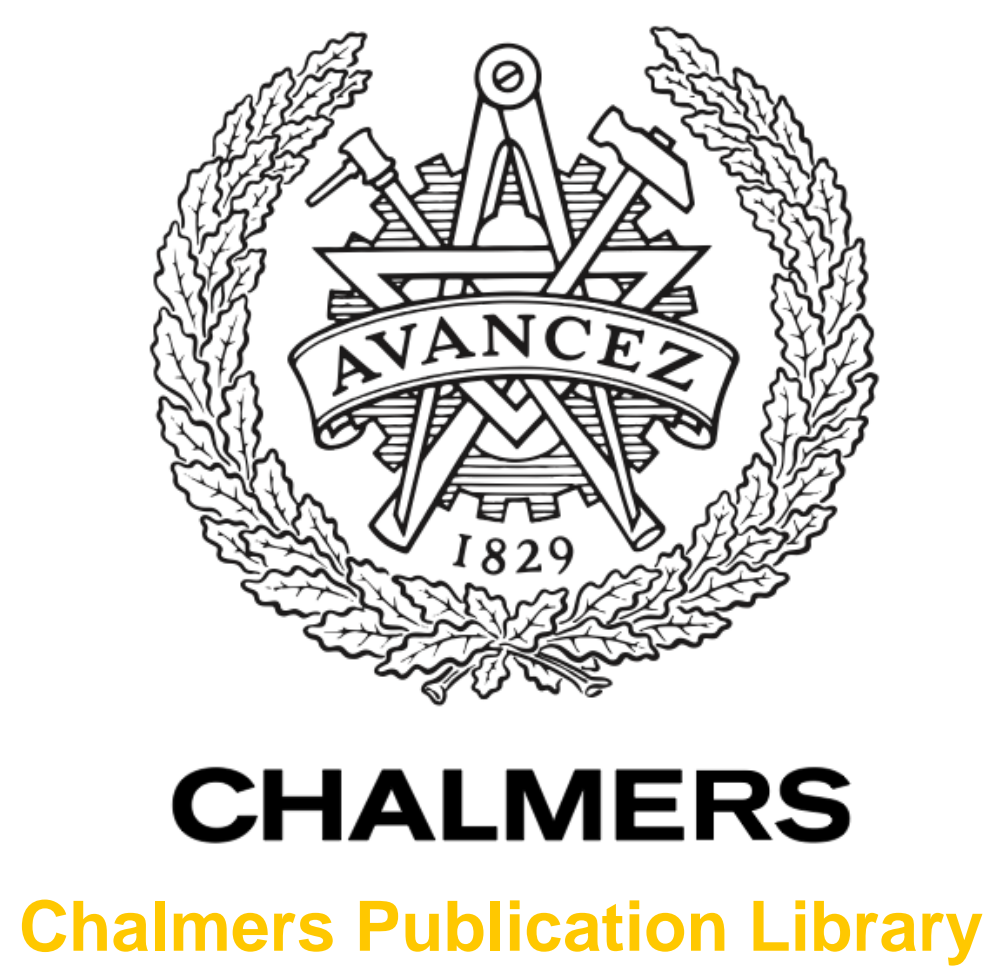

Finline-integrated cold electron bolometer

This document has been downloaded from Chalmers Publication Library (CPL). It is the author's version of a work that was accepted for publication in:

Proceedings of SPIE - The International Society for Optical Engineering (ISSN: 0277-786X)

Citation for the published paper:

Otto, E. ; Tarasov, M. ; Grimes, P. (2010) "Finline-integrated cold electron bolometer".

Proceedings of SPIE - The International Society for Optical Engineering, vol. 7741

http://dx.doi.org/10.1117/12.858263

Downloaded from: http://publications.lib.chalmers.se/publication/126684

Notice: Changes introduced as a result of publishing processes such as copy-editing and formatting may not be reflected in this document. For a definitive version of this work, please refer to the published source. Please note that access to the published version might require a subscription. 


\title{
Finline-integrated cold electron bolometer
}

\author{
Ernst Otto $^{a}$, Mikhail Tarasov ${ }^{b, c}$, Paul K. Grimes ${ }^{a}$, Natalia S. Kaurova ${ }^{b, d}$, Hannes Kuusisto ${ }^{b}$, Leonid \\ S. Kuzmin ${ }^{b}$ and Ghassan Yassin ${ }^{a}$ \\ ${ }^{a}$ Oxford University, Oxford, United Kingdom; \\ ${ }^{b}$ Chalmers University of Technology, Gothenburg, Sweden; \\ ${ }^{c}$ Kotel'nikov Institute of Radio Engineering and Electronics, Moscow, Russia; \\ ${ }^{d}$ Moscow State Pedagogical University, Moscow, Russia
}

\begin{abstract}
The Cold-Electron Bolometer (CEB) is a sensitive millimetre-wave detector which is easy to integrate with superconducting planar circuits. CEB detectors have other important features such as high saturation power and very fast response. We have fabricated and tested CEB detectors integrated across the slot of a unilateral finline on a silicon substrate. Bolometers were fabricated using two fabrication methods: e-beam direct-write trilayer technology and an advanced shadow mask evaporation technique. The CEB performance was tested in a $\mathrm{He}^{3}$ sorption cryostat at a bath temperature of $280 \mathrm{mK}$. DC I-V curves and temperature responses were measured in a current bias mode, and preliminary measurements of the optical response were made using an IMPATT diode operating at $110 \mathrm{GHz}$. These tests were conducted by coupling power directly into the finline chip, without the use of waveguide or feedhorns. For the devices fabricated in standard direct-write technology, the bolometer dark electrical noise equivalent power is estimated to be about $5 \times 10^{-16} \mathrm{~W} / \sqrt{\mathrm{Hz}}$, while the dark NEP value for the shadow mask evaporation technique devices is estimated to be as low as $3 \times 10^{-17} \mathrm{~W} / \sqrt{\mathrm{Hz}}$.
\end{abstract}

\section{INTRODUCTION}

The Cold Electron Bolometer ${ }^{1}$ (CEB) is a sensitive millimetre-wave detector with the potential to achieve high sensitivity in conjunction with high saturation power and fast response times. The CEB is easy to integrate with superconducting planar circuits and antennas, and can be used with both SQUID ${ }^{1}$ and $\mathrm{FET}^{2}$ based readout systems.

In this paper we present DC measurements and preliminary optical measurements of two cold electron bolometers with SIN tunnel junctions and normal metal absorbers, one fabricated using e-beam direct-write patterning of trilayer SIN junctions and the absorber, ${ }^{3,4}$ and the other using an advanced shadow mask evaporation technique. ${ }^{5}$ The CEBs are designed for operation at $70-110 \mathrm{GHz}$, and are deposited across unilateral finlines on a silicon substrate. For full optical testing, the finline chip will be mounted in WR-10 waveguide and coupled to the incoming signal via a smooth-wall feedhorn.

The CEB devices considered here consist of a small normal metal absorber coupled to superconducting electrodes via SIN tunnel junctions at each end of the absorber (fig. 1). RF power from the antenna is capacitively coupled through the SIN tunnel junctions into the absorber. When a photon is absorbed in the normal metal, an electron is excited. This excitation is rapidly converted via electron-electron interactions into a heating of the whole absorber electron system. When the CEB is biased just below the gap voltage of the SIN junctions, the hottest electrons in the absorber rapidly tunnel through the SIN junctions, giving an increase in bias current, as well as cooling the absorber. ${ }^{6}$ Thermal isolation of the absorber's electron system is provided by the relatively long electron-phonon interaction time in the absorber $\left(\tau_{e-p h} \sim 10^{-5} \mathrm{~s}\right)$, compared to the time taken for electrons to tunnel through the SIN junctions $\left(\tau \sim 10^{-8} \mathrm{~s}\right)$.

An important feature of the CEB is cooling of the electron system in the absorber by the SIN tunnel junctions, which provides electrothermal feedback. ${ }^{7}$ Incoming power is removed from the absorber, delaying the onset of saturation and thus increasing the dynamic range of the detector. The time constant of the CEB can be reduced by the loop gain of negative electrothermal feedback to $\sim 10 \mathrm{~ns}$.

Further author information: (Send correspondance to PKG)

Ernst Otto: E-mail otto@astro.ox.ac.uk

Paul K. Grimes: E-mail p.grimes1@ physics.ox.ac.uk, Telephone: +44 (0)1865 273304

Millimeter, Submillimeter, and Far-Infrared Detectors and Instrumentation for Astronomy V, edited by Wayne S. Holland, Jonas Zmuidzinas, Proc. of SPIE Vol. 7741, 77411A

(c) 2010 SPIE $\cdot$ CCC code: $0277-786 X / 10 / \$ 18 \cdot$ doi: $10.1117 / 12.858263$

Proc. of SPIE Vol. $774177411 \mathrm{~A}-1$ 

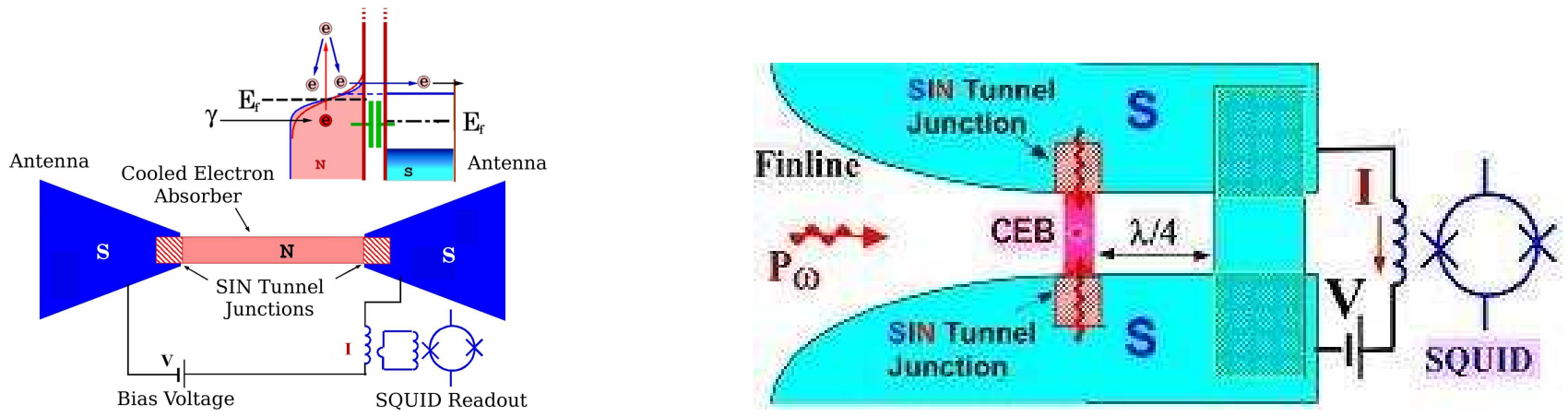

Figure 1. Schematic of the operation and design of an SIN based CEB in a unilateral finline, read in the voltage biased mode by a SQUID current amplifier.
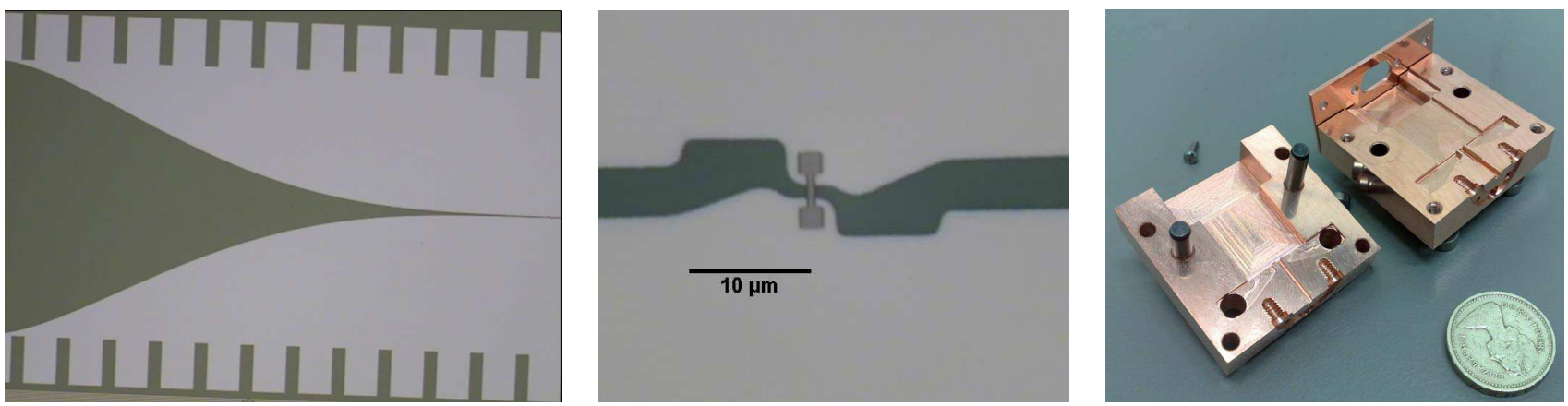

Figure 2. Microscope photographs of the finline taper (left), and CEB device (centre) fabricated by the direct-write process. (right) The split waveguide detector block. The CEB device will be mounted in the WR-10 waveguide at the lower right of block as shown.

The CEB can be operated in either a constant current or constant voltage bias mode. In both cases, the dynamic impedance of the device at the operating point must be matched to readout circuit to achieve the best noise performance. For SIN based CEBs, with a normal metal absorber impedance matched to the antenna, the dynamic impedance is dominated by the sub-gap resistance of the SIN tunnel junctions.

\section{BOLOMETER DESIGN}

We have fabricated finline CEB devices using two techniques, electron-beam direct-write devices using SIN junctions fabricated in trilayers, and devices fabricated using an advanced shadow mask evaporation technique.

The first set of devices were fabricated by first depositing gold contact pads and wires, followed by deposition of the finlines. Both of these layers are fabricated using e-beam exposure and thermal evaporation. The CEB structure is then fabricated in the finline slot using the trilayer process proposed by L. Kuzmin (fig. 3). ${ }^{3}$ One disadvantage of this technique is that the absorber must be made thick enough to step up onto the top contacts of the tunnel junctions. This places a lower limit on the thickness and hence volume of the absorber.

The second set of devices are fabricated using an advanced shadow mask evaporation technique. ${ }^{5}$ In this approach, the absorber is deposited prior to the tunnel junctions, and then oxidized. The superconducting electrodes are then deposited with the sample remaining in vacuum throughout. This results in a high quality tunnel barrier and an absorber with very small volume, increasing the sensitivity of the device.

Both CEB devices are fabricated across a $5 \mu \mathrm{m}$ wide slotline fabricated on a $240 \mu \mathrm{m}$ thick silicon substrate, and fed by a unilateral finline taper. The absorber dimensions are chosen to give an absorber resistance equal to the slotline impedance of $50 \Omega$, resulting in an $50: 50$ copper/chromium absorber $4 \mu \mathrm{m}$ long by $0.5 \mu \mathrm{m}$ wide and $50 \mathrm{~nm}$ thick for devices fabricated using the direct-write technique. The SIN tunnel junctions are made from an $\mathrm{Al}-\mathrm{Al}_{2} \mathrm{O}_{3}-\mathrm{Cu}$ trilayer, covered in a thin layer of gold to prevent oxidation of the copper during processing. Devices fabricated by the shadow mask technique ${ }^{5}$ 
a)

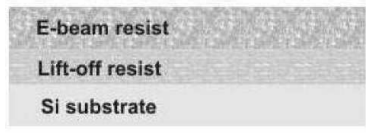

b)

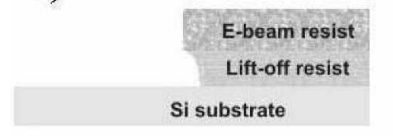

c)

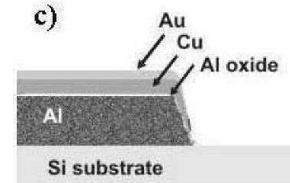

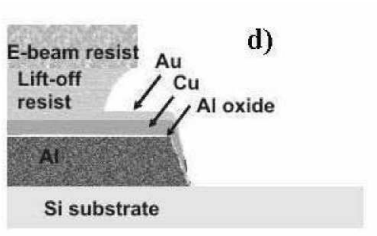

e)

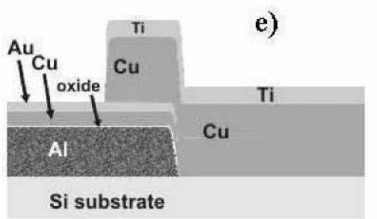

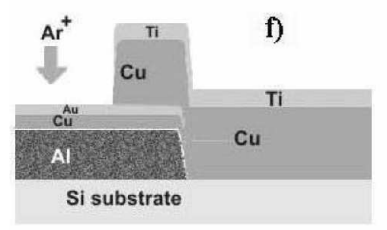

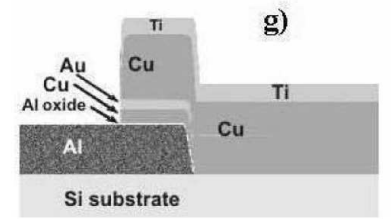

Figure 3. Direct write fabrication process. (a \& b) The resist mask for the trilayer is defined by e-beam lithography. (c) The $\mathrm{Al}-\mathrm{Al}_{2} \mathrm{O}_{3}-\mathrm{Cu}$ trilayer is deposited, covered by a gold protection layer, and lifted-off. (d) E-beam lithography is used to define the absorber area. (e) The copper/chromium absorber is deposited and lifted-off, covered by a thin titanium layer to protect the absorber during ion beam etching. (f) The gold and copper top electrode is removed from the exposed area of the trilayer by ion beam etching, leaving SIN junctions under the absorber contacts only $(\mathrm{g})$.
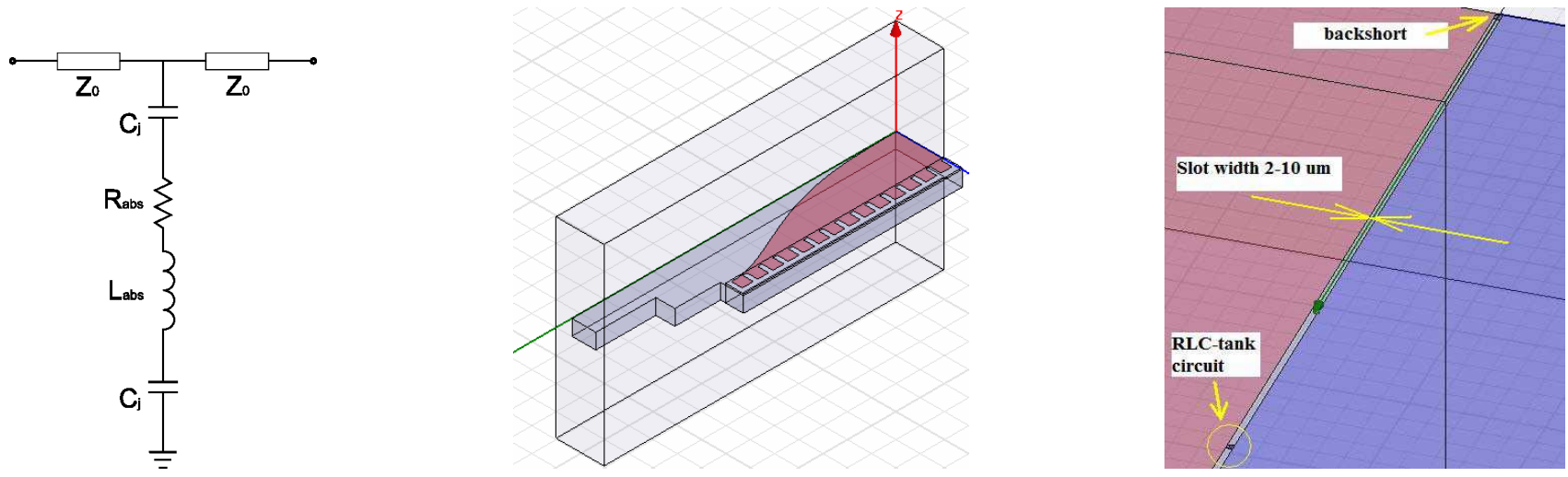

Figure 4. Equivalent lumped element model of the CEB used in RF simulations (left), and the HFSS model used to calculate the coupling to the CEB (centre, right). The central image shows the finline taper with serrations and substrate extending into the chip support slot. Only half of the structure is shown, as the symmetry of the structure is exploited to speed up the simulation. The right image shows the detail of CEB device (represented by the RLC circuit), slotline and back-short.

use non-superconducting aluminium absorbers $2 \mu \mathrm{m}$ long by $120 \mathrm{~nm}$ wide and $20 \mathrm{~nm}$ thick. The junction area of $\sim 2 \mu \mathrm{m}^{2}$ results in a normal resistance of $\sim 400 \Omega$ per junction, giving a final device normal resistance of $800-1000 \Omega$. DC signals are carried to the CEB by gold contact wiring and the finline electrodes.

Power is coupled into the slotline via a unilateral finline taper, designed using an optimum taper method. ${ }^{8}$ The detector chip is mounted in a WR-10 waveguide, supported by grooves in the waveguide wall. Quarter-wavelength serrations are fabricated on the edge of finline chip to suppress higher-order modes in the groove. The front of the detector chip is shaped into either a shallow triangular taper by dicing, or a two-stage stepped transformer by dry etching, to match the incoming signal to the loaded waveguide.

The RF impedance of the CEB device has three components; the resistance of the absorber $R_{a b s}$, the geometric inductance of the absorber strip $L_{a b s}$, and the capacitance of the two SIN tunnel junctions $C_{j}$. Since the CEB device is much smaller than the operating wavelength, we model the device as a lumped element RLC series circuit, shown in fig. 4 (left). A capacitively coupled back-short is deposited across the slotline at a distance optimized to tune out the reactance of the CEB device and to maximise coupling to the absorber (fig. 4 (centre and right)). The performance of the entire chip, including substrate matching, finline taper, serrations, back-short and CEB device has been simulated using Ansoft HFSS 


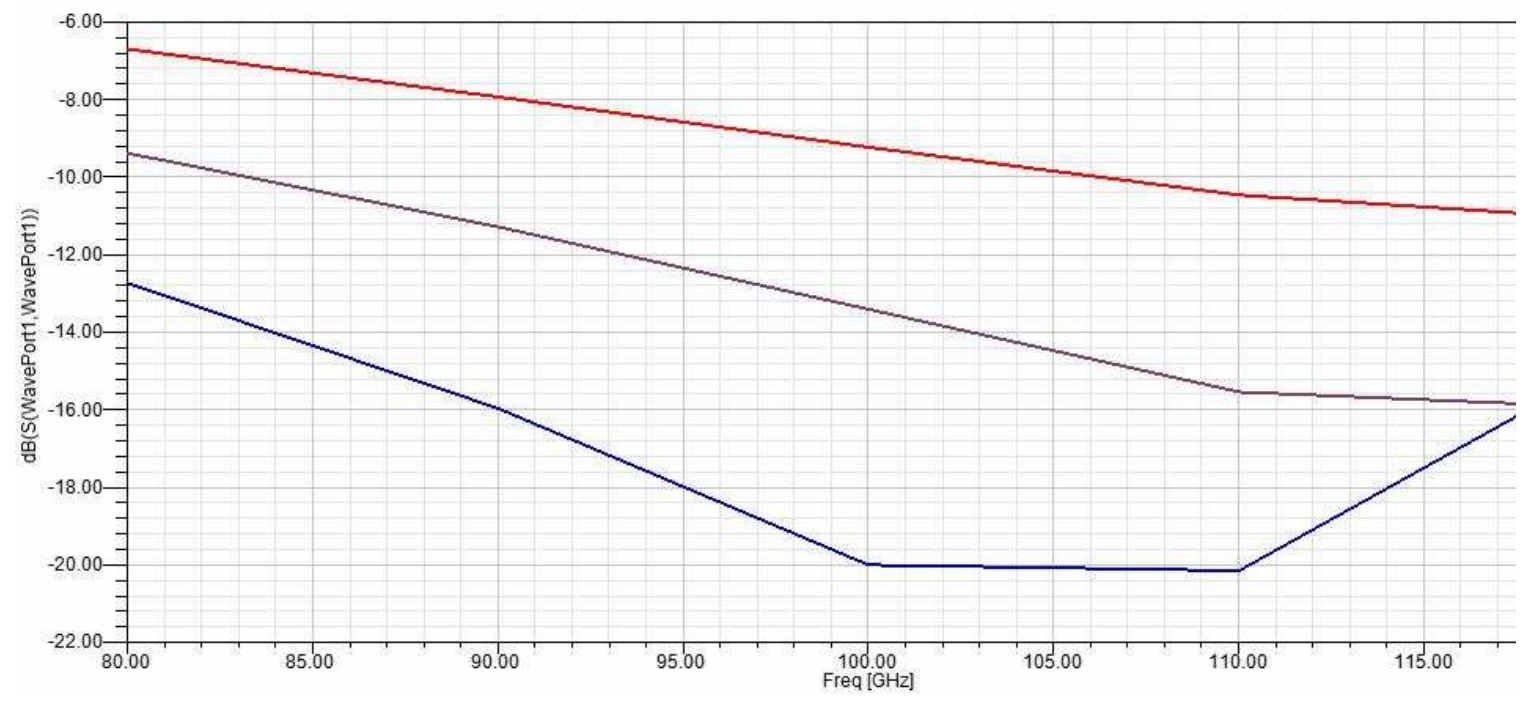

Figure 5. Return loss calculated for the direct-write CEB, with a slot width of $5 \mu \mathrm{m}$, junction capacitance of $200 \mathrm{fF}$, absorber resistance of $50 \Omega$ and geometric inductance $2.6 \mathrm{pH}$. The back-short distance is varied between $220 \mu \mathrm{m}$ (red) to $250 \mu \mathrm{m}$ (blue).

(fig. 5). This simulation was also used to optimized the the back-short distance to give the best return loss across the WR-10 waveguide band.

\section{CEB SENSITIVITY AND NOISE}

The operation of the cold electron bolometer has been studied by Golubev and Kuzmin. ${ }^{9}$ The heat balance equation for the CEB is

$$
P_{0}+\delta P(t)=C_{\Lambda} \frac{d T}{d t}+P_{S I N}\left(V, T_{e}, T_{p h}\right)+\frac{V^{2}}{R_{j}}+I^{2} R_{a b s}+\Sigma \Lambda\left(T_{e}^{5}-T_{p h}^{5}\right),
$$

where $P_{0}$ is the background power on the bolometer, $\delta P(t)$ is the changing power on the bolometer, $C_{\Lambda}$ is heat capacity of the absorber and $P_{S I N}$ is the heat flow through the SIN tunnel junctions. The $\frac{V^{2}}{R_{j}}$ term is the heating of the bolometer by the bias current through the tunnel junction's dynamic resistance $R_{j}$, and the $I^{2} R_{a b s}$ term is Joule heating of the absorber resistance $R_{a b s}$ by the bias current $I$. The final $\Sigma \Lambda\left(T_{e}^{5}-T_{p h}^{5}\right)$ term is the heat transferred by electron-phonon interactions in the absorber, where $\Lambda$ is the volume of absorber and $\Sigma$ is a material constant.

Taking the time dependent part of (1) we obtain

$$
\frac{\delta P(t)}{\delta T}=\frac{\partial P_{S I N}}{\partial T}+5 \Sigma \Lambda T_{e}^{4}+i \omega C_{\Lambda}=G_{S I N}+G_{e-p h}+i \omega C_{\Lambda}=G_{e f f}
$$

where the first term of (2), $G_{S I N}=\frac{\partial P_{S I N}}{\partial T}$, is the thermal conductance of the SIN tunnel junction. This term gives the negative electrothermal feedback (ETF); when large, this term reduces the temperature response $\delta T$ because the cooling power compensates for the change in signal power in the bolometer. The second term, $G_{e-p h}=5 \Sigma \Lambda T_{e}^{4}$, is the electronphonon thermal conductance in the absorber, and the third term is due to the thermal capacity of the absorber. The sum of these conductances is the effective thermal conductance $G_{\text {eff }}$.

In the constant voltage biased case, the response of the bolometer is characterized by the current responsivity $S_{I}$, which is given by the ratio of the current change $\partial I$ due to a temperature change in the absorber $\partial T$, and the change in power load required to give this temperature change

$$
S_{I}=\frac{\partial I_{\omega}}{\partial P_{\omega}}=\frac{\frac{\partial I}{\partial T}}{G_{S I N}+G_{e-p h}+i \omega C_{\Lambda}}=\frac{\frac{\partial I}{\partial T} L}{G_{S I N}(L+1)(1+i \omega \tau)}
$$



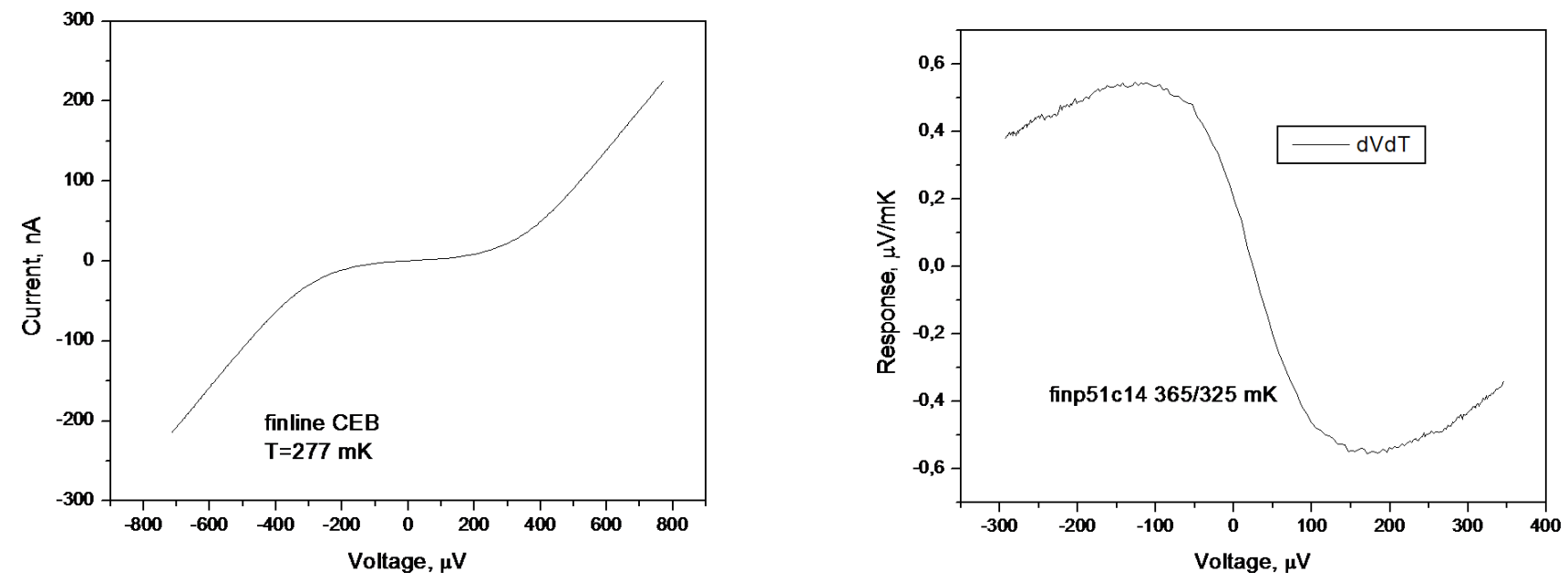

Figure 6. Current-voltage characteristic and voltage response for direct-write device finp51c4 .

where $L=G_{S I N} / G_{e-p h} \gg 1$ is the ETF gain and $\tau=C_{\Lambda} / G_{e-p h}(L+1)=\tau_{0} /(L+1)$ is the effective time constant. The intrinsic time constant is given by $\tau_{0}=C_{\Lambda} / G_{e-p h}$ and is typically around $10 \mu \mathrm{s}$ at $100 \mathrm{mK}$. The constant current responsivity, $S_{V}=\frac{\partial V_{\omega}}{\partial P_{\omega}}$ can be calculated in similar way, with $\partial V / \partial T$ replacing $\partial I / \partial T$.

Noise in the CEB can be divided into three major uncorrelated contributions:

$$
N E P_{\text {tot }}^{2}=N E P_{e-p h}^{2}+N E P_{S I N}^{2}+N E P_{a m p}^{2}
$$

where $N E P_{e-p h}$ is the thermal fluctuation noise associated with the electron-phonon interaction, given by $N E P_{e-p h}=$ $10 k_{B} \Sigma \Lambda\left(T_{e}^{6}+T_{p h}^{6}\right) . N E P_{a m p}$ is the noise due to the readout circuit. In voltage biased mode, this is given by $N E P_{a m p}=\frac{\sqrt{i^{2}}}{S_{I}}$, where $\sqrt{\tilde{i}^{2}}$ is the input referenced current noise of the readout system, and $S_{I}$ is the current responsivity of the CEB.

The noise due to the SIN tunnel junction has two anti-correlated components, due to shot noise from the current flow through the junction, and fluctuations of heat flow through the junction. This gives the total noise due to each SIN junction as $N E P_{S I N}^{2}=\delta P_{\omega}^{2}-2 \frac{\delta P_{\omega} \delta I_{\omega}}{S_{I}}+\frac{\delta I_{\omega}^{2}}{S_{I}^{2}}$.

\section{MEASUREMENTS}

Testing of both devices was carried out at Chalmers University of Technology using an Oxford Instruments HELIOX-ACV helium-3 sorption cryostat, which can reach a base temperature of $280 \mathrm{mK}$. In order to keep the optical power load on the detectors to acceptable levels, two low-pass filters with cut-off frequencies of $33 \mathrm{~cm}^{-1}$ and $100 \mathrm{~cm}^{-1}$ and two neutral density filters with $10 \mathrm{~dB}$ attenuation each were mounted over the windows in the radiation shields inside the cryostat.

DC current-voltage characteristics (I-V curves) of both CEB devices were measured in the fixed current bias mode, using a differential bias circuit with room temperature amplifiers and bias resistors. This circuit uses a driver circuit with bias resistance switchable in a range from $100 \mathrm{k} \Omega$ to $10 \mathrm{G} \Omega$, and AD743 BiFET based first stage amplifiers with input voltage noise of around $4 \mathrm{nV} / \sqrt{\mathrm{Hz}}$. I-V curves were taken at several bath temperatures between $286 \mathrm{mK}$ and $356 \mathrm{mK}$.

The current and voltage temperature responses, $S_{I / T}=\frac{\partial I}{\partial T}$ and $S_{V / T}=\frac{\partial V}{\partial T}$, of each CEB is calculated by comparing the I-V curves as the bath temperature is varied. The measured I-V curve and voltage response for the direct-write device are shown in fig. 6. The measured I-V curves and voltage response for the shadow mask evaporation device are shown in fig. 7. Fig. 8 (left) shows the current responsivity for the shadow mask device.

Noise measurements were made using a lock-in amplifier referenced to a signal generator to make a spot measurement of the noise power at the voltage output of the bias system at $127 \mathrm{~Hz}$, using a $24 \mathrm{~dB} /$ oct filter. The measured voltage noise for the direct-write device is shown as a function of bias voltage in fig. 8 (right). 

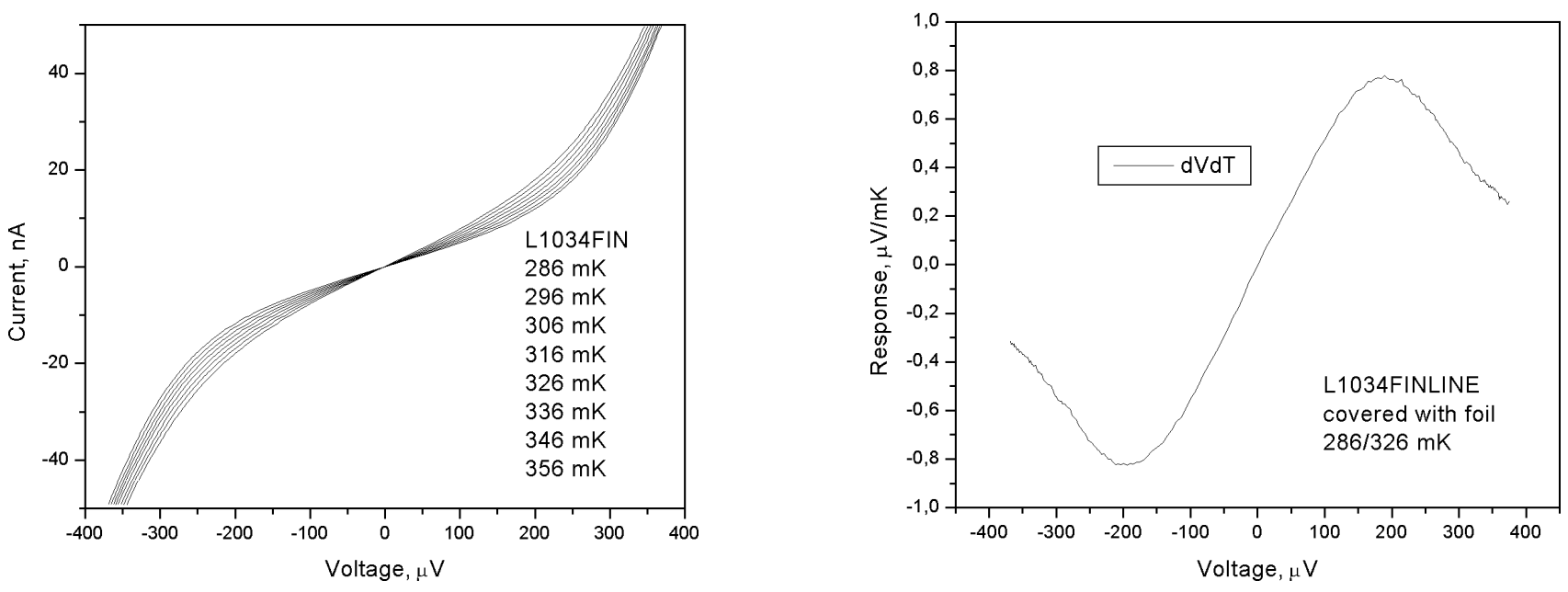

Figure 7. Current-voltage characteristic as a function of temperature and voltage response for shadow mask device L1034FIN. The most non-linear I-V curve corresponds to the lowest temperature.
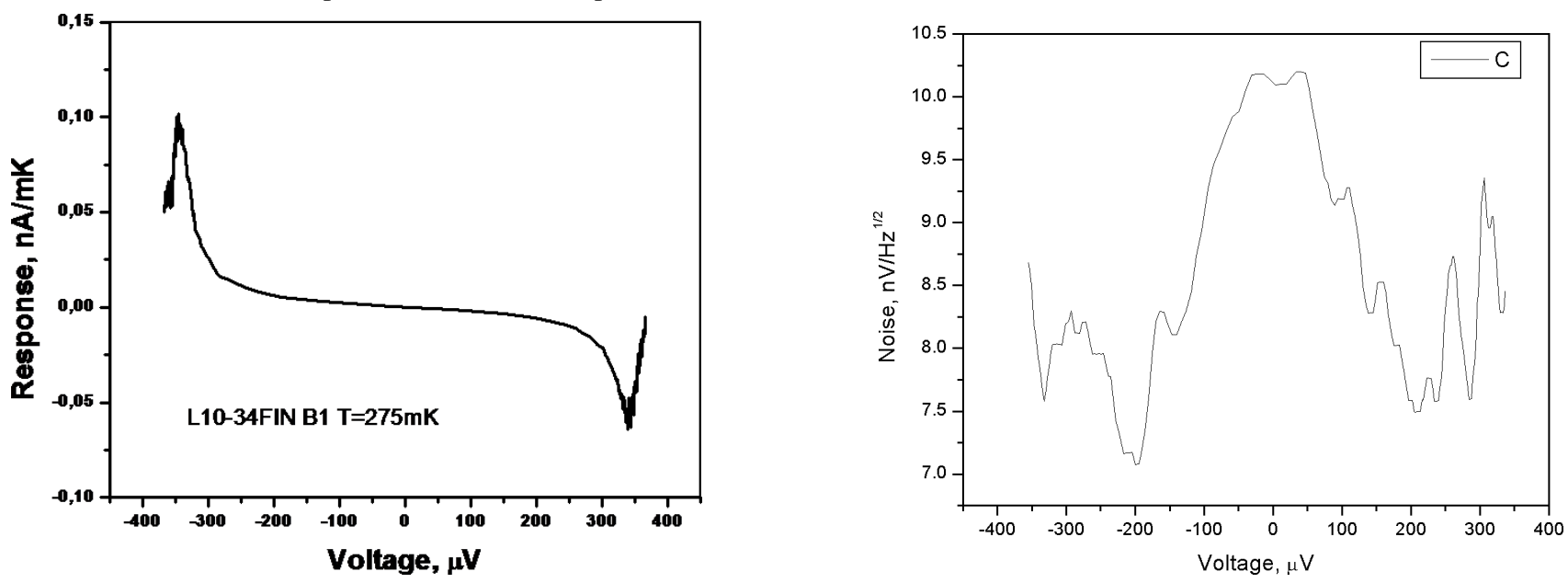

Figure 8. Current responsivity (left) for shadow mask device L1034FIN, and measured voltage noise at $127 \mathrm{~Hz}$ (right) for the direct write device in the current bias scheme.

To make preliminary optical response measurements, the finline chip was mounted on a metal sample holder facing the cryostat window. A small amount of optical power will be coupled into finline even when not mounted in the waveguide, as it behaves somewhat like a Vivaldi antenna, loaded on one side by the dielectric of the substrate. Optical power was provided by a $110 \mathrm{GHz}$ IMPATT diode and coupled via a feed-horn. The output of the IMPATT diode was modulated at $127 \mathrm{~Hz}$ and the bolometer response detected via a lock-in amplifier, at three levels of signal power. The measured optical response is shown in fig. 9 (centre).

\section{ANALYSIS OF RESULTS}

Using the experimental data above for the direct-write device, we can estimate the dark NEP in the current bias mode. The voltage response is $S_{V}=\frac{d V}{d P}=\frac{d V}{d T} \frac{1}{G}$. The thermal conductance $G$ is made up of two major components, due to the electron-phonon interaction $\left(G_{e-p h}\right)$ and to the energy carried by the bias current through the tunnel junctions $\left(G_{S I N}\right)$. For a clean copper absorber $500 \mathrm{~nm}$ wide by $4 \mu \mathrm{m}$ long and $50 \mathrm{~nm}$ thick, with material constant $\Sigma=3 \times 10^{9} \mathrm{Wm}^{-3} \mathrm{~K}^{-5}$ and at a bath temperature of $280 \mathrm{mK}, G_{e-p h}=5 \Sigma \Lambda T_{e}^{4}=1.84 \times 10^{-11} \mathrm{~W} / \mathrm{K}$. For two $2 \mu \mathrm{m}^{2} \mathrm{SIN}$ tunnel junctions and a bias current of $20 \mathrm{nA}$, the junction thermal conductance is $G_{S I N}=1.6 \times 10^{-12} \mathrm{~W} / \mathrm{K}$, giving a net thermal conductance of $G \sim 2 \times 10^{-11} \mathrm{~W} / \mathrm{K}$. 

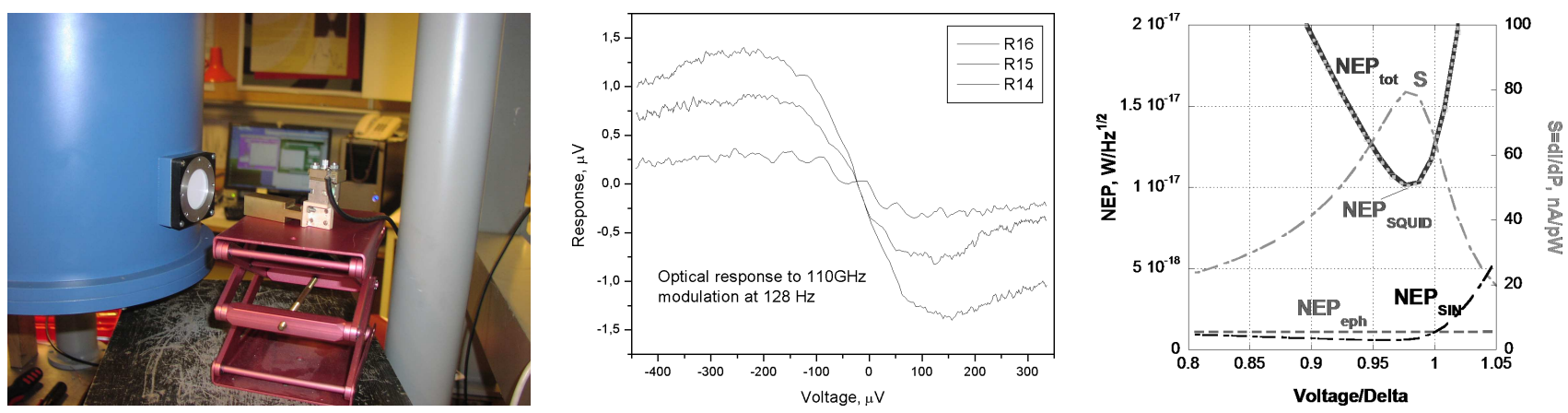

Figure 9. Preliminary optical response measurement setup (left) and measured response (centre) at three power levels. Right: Expected noise and responsivity for an improved CEB based on the measured shadow-mask device, and read by a SQUID current amplifier.

Taking the measured voltage-temperature response for the direct-write device of $0.5 \mathrm{mV} / \mathrm{K}$, and the maximum measured voltage noise of the bolometer and readout system, $10 \mathrm{nV} / \sqrt{\mathrm{Hz}}$, we estimate the noise in the current bias mode to be $N E P_{\text {iBias }}=5 \times 10^{-16} \mathrm{~W} / \sqrt{\mathrm{Hz}}$.

For the device fabricated using the shadow mask evaporation technique, we carry out a similar calculation for operation in the voltage biased mode. In this case the relevant responsivity is the current responsivity $S_{I}=\frac{d I}{d P}=\frac{d I}{d T} \frac{1}{G}$. The SIN tunnel junctions are $3 \times 0.6 \mu \mathrm{m}^{2}$, with a small aluminium absorber $\left(0.12 \times 3 \mu \mathrm{m}^{2}\right.$, thickness $\left.10-20 \mathrm{~nm}\right)$ and $\Sigma=2 \times$ $10^{9} \mathrm{Wm}^{-3} \mathrm{~K}^{-5}$. At a temperature of $280 \mathrm{mK}$, this gives an electron-phonon conductance of $G_{e-p h}=1.2 \times 10^{-12} \mathrm{~W} / \mathrm{K}$. At a bias current of $20 \mathrm{nA}$, the SIN tunnel junction conductance is $G_{S I N}=1.6 \times 10^{-12} \mathrm{~W} / \mathrm{K}$.

We assume that in the voltage bias mode, the current through this device will be read by a SQUID with a typical current noise around $\sqrt{\tilde{i^{2}}}=1 \mathrm{pA} / \sqrt{\mathrm{Hz}}$. At a bias current of $20 \mathrm{nA}$, the noise contribution from shot noise in the two tunnel junctions is $\sqrt{i_{\text {shot }}^{\sim}}=\sqrt{e I}=55 \mathrm{fA} / \sqrt{\mathrm{Hz}}$.

Using the measured current response of the bolometer, $S_{I / T}=\frac{d I}{d T}=0.1 \mathrm{nA} / \mathrm{mK}$, we anticipate that the dark NEP in the voltage bias scheme will be $N E P_{v \text { Bias }}=\frac{\sqrt{\tilde{i}^{2}}}{S_{I}}=\frac{\sqrt{i^{2}}}{S_{I / T}} G=3 \times 10^{-17} \mathrm{~W} / \sqrt{\mathrm{Hz}}$.

We expect improvements in the fabrication and design of this device and its bias circuit to yield improved current responsivity. Fig. 9 (right) shows the calculated NEP and responsivity anticipated for a similar device with a responsivity a few times better than our estimated value for the measured device.

\section{CONCLUSIONS}

We have fabricated and tested SIN based Cold Electron Bolometers coupled to unilateral finline tapers, using two fabrication techniques. The direct-write electron-beam lithography technique is limited to devices with relatively large absorbers that are more suited for current biased operation using FET based readout, while the advanced shadow mask evaporation technique can produce much smaller absorbers giving more sensitive devices suitable for voltage biased operation with SQUID readout. Using the measured voltage-temperature and current-temperature response of the devices, with estimates of the thermal conductivity we have estimated the dark NEPs of these devices as $5 \times 10^{-16} \mathrm{~W} / \sqrt{\mathrm{Hz}}$ for the current biased direct-write device, at a spot noise frequency of $127 \mathrm{~Hz}$, and $3 \times 10^{-17} \mathrm{~W} / \sqrt{\mathrm{Hz}}$ for the shadow mask device in the voltage bias mode when read out by a SQUID.

We have also made initial optical response measurements of the bolometers using an $110 \mathrm{GHz}$ IMPATT diode. In this case the finline chip was directly illuminated by the source, resulting in poor power coupling. Despite this, a strong response to optical power was seen, indicating the sensitivity of these bolometers.

We are now concentrating on developing the measurement system in Oxford to make a more comprehensive noise measurements of both differential and single-ended FET based bias circuits, and preparing to make calibrated optical response measurements using cryogenic temperature controlled blackbody loads coupled to the finline devices via feedhorns and waveguide. 
Better matching to FET based readout circuits, and hence lower noise, may be obtained ${ }^{10}$ by the use of weakly superconducting absorbers in the place of our normal metal absorbers, and/or the use of parallel/series arrays of devices, in which the absorbers are placed in parallel across the antenna for AC input, but are biased and read out in series. We are currently also designing finline devices with small arrays with both superconducting and normal absorbers.

\section{REFERENCES}

1. Kuzmin, L.S. and Fominsky, M. and Kalabukhov, A. and Golubev, D. and Tarasov, M., "Capacitively coupled hotelectron nanobolometer with SIN tunnel junctions," in Society of Photo-Optical Instrumentation Engineers (SPIE) Conference Series, T. G. Phillips \& J. Zmuidzinas, ed., 4855, pp. 217-226, Feb. 2003.

2. L. Kuzmin and P. Mauskopf, "Superconducting cold-electron bolometers with JFET readout for OLIMPO balloon telescope," in Sixteenth International Symposium on Space Terahertz Technology, held May 2-4, 2005 at Chalmers University of Technology. Gothenburg, Sweden., pp. 549-554, May 2005.

3. E. Otto, M. Tarasov, and L. Kuzmin, "Direct-write trilayer technology for $\mathrm{Al}-\mathrm{Al}_{2} \mathrm{O}_{3}-\mathrm{Cu}$ superconductor-insulatornormal metal tunnel junction fabrication," Journal of Vacuum Science Technology B: Microelectronics and Nanometer Structures 25, p. 1156, 2007.

4. E. Otto, M. Tarasov, G. Pettersson, D. Gustavsson, and L. Kuzmin, "An array of $100 \mathrm{Al}-\mathrm{Al}_{2} \mathrm{O}_{3}-\mathrm{Cu}$ SIN tunnel junctions in direct-write trilayer technology," Superconductor Science Technology 20, pp. 1155-1158, Dec. 2007.

5. L. S. Kuzmin, "Self-aligned shadow-evaporation technology for large area tunnel junctions and nanoabsorbers," PCT Patent, to be filed, 2010.

6. L. Kuzmin, I. Agulo, M. Fominsky, A. Savin, and M. Tarasov, "Optimization of electron cooling by SIN tunnel junctions," Superconductor Science Technology 17, p. 400, May 2004.

7. L. Kuzmin, "Ultimate cold-electron bolometer with strong electrothermal feedback," in Society of Photo-Optical Instrumentation Engineers (SPIE) Conference Series, C. M. Bradford, P. A. R. Ade, J. E. Aguirre, J. J. Bock, M. Dragovan, L. Duband, L. Earle, J. Glenn, H. Matsuhara, B. J. Naylor, H. T. Nguyen, M. Yun, \& J. Zmuidzinas, ed., 5498, pp. 349-361, Oct. 2004.

8. C. North, G. Yassin, and P. Grimes, "Rigorous analysis and design of finline tapers for high performance millimetre and submillimetre detectors," in Seventeenth International Symposium on Space Terahertz Technology, held May 10-12, 2006 at Observatoire de Paris, LERMA. Paris, France., p.284-287, pp. 284-287, May 2006.

9. D. Golubev and L. Kuzmin, "Nonequilibrium theory of a hot-electron bolometer with normal metal-insulatorsuperconductor tunnel junction," Journal of Applied Physics 89, pp. 6464-6472, June 2001.

10. L. Kuzmin, "A parallel/series array of superconducting cold-electron bolometers with SIS' tunnel junctions," in Society of Photo-Optical Instrumentation Engineers (SPIE) Conference Series, 7020, Aug. 2008. 